\title{
Phytoprotection
}

\section{Note sur l'aire de distribution et l'importance du Dacnusa dryas, un parasite introduit au Québec pour lutter contre l'agromyze de la lyzerne (Agromyza frontella)}

\author{
M. Letendre, M. O'c. Guibord, D.G. Harcourt et J.C. Guppy
}

Volume 72, numéro 1, 1991

URI : https://id.erudit.org/iderudit/706001ar

DOI : https://doi.org/10.7202/706001ar

Aller au sommaire du numéro

Éditeur(s)

Société de protection des plantes du Québec (SPPQ)l

ISSN

0031-9511 (imprimé)

1710-1603 (numérique)

Découvrir la revue

Citer cet article

Letendre, M., Guibord, M., Harcourt, D. \& Guppy, J. (1991). Note sur l'aire de distribution et l'importance du Dacnusa dryas, un parasite introduit au Québec pour lutter contre l'agromyze de la lyzerne (Agromyza frontella).

Phytoprotection, 72(1), 33-35. https://doi.org/10.7202/706001ar
Résumé de l'article

De 1978 à 1980, le Dacnusa dryas, un hyménoptère d'origine européenne parasitant les larves et les pupes de l'agromyze de la luzerne, Agromyza frontella, fut relâché successivement dans deux localités du Québec. Une étude détaillée de son aire de distribution effectuée en 1986 démontre que l'insecte s'est dispersé dans les douze régions agricoles du Québec. Suite à sa naturalisation, les populations d'agromyze ont diminué à des niveaux sous-économiques. 


\title{
Note sur l'aire de distribution et l'importance du Dacnusa dryas, un parasite introduit au Québec pour lutter contre l'agromyze de la luzerne (Agromyza frontella)
}

\author{
M. Letendre et M. O'c. Guibord \\ Direction de la recherche et du développement, \\ Ministère de l'Agriculture, des Pêcheries et de l'Alimentation du Québec, \\ Sainte-Foy (Québec) Canada G1P 3W8. Contribution No 377

\section{D.G. Harcourt et J.C. Guppy} \\ Centre des recherches phytotechniques, Direction générale de la recherche, \\ Agriculture Canada, Ottawa (Ontario) Canada K1A 0C6. Contribution No PRC-1275
}

(Reçu 1990-06-21; accepté 1991-03-07)

\begin{abstract}
De 1978 à 1980, le Dacnusa dryas, un hyménoptère d'origine européenne parasitant les larves et les pupes de l'agromyze de la luzerne, A gromyza frontella, fut relâché successivement dans deux localités du Québec. Une étude détaillée de son aire de distribution effectuée en 1986 démontre que l'insecte s'est dispersé dans les douze régions agricoles du Québec. Suite à sa naturalisation, les populations d'agromyze ont diminué à des niveaux souséconomiques.
\end{abstract}

Letendre, M., M. O'c. Guibord, D.G. Harcourt et J.C. Guppy. 1991. Note sur l'aire de distribution et l'importance du Dacnusa dryas, un parasite introduit au Québec pour lutter contre l'agromyze de la luzerne (Agromyza frontella). PHYTOPROTECTION 72: 33- 35.

Dacnusa dryas, a European larval-pupal parasitoid of the alfalfa blotch leafminer, Agromyza frontella, was successively released in two areas of Quebec from 1978 to 1980. A detailed survey in 1986 shows that it has become established in all 12 agricultural regions. Coincident with its successful colonization, populations of the host have declined to subeconomic levels.

L'agromyze de la luzerne, Agromyza frontella (Rond.), a envahi les provinces du centre et de l'est du Canada au début des années 1970. Dans le but de réduire les populations de cet insecte, le parasite européen Dacnusa dryas (Nixon) a été introduit au Québec lors de quatre lâchers effectués de 1978 à 1980. Pour ce, des colonies totalisant 480 individus furent obtenues de monsieur R. Hendrickson du Laboratoire de recherche sur les insectes bénéfiques, Ministère de l'Agriculture des États-Unis à Newark, Delaware. Les introductions eurent lieu à Saint-Hyacinthe, avec deux lâchers en 1978 et un en 1979, et à SaintAugustin-de-Desmaures, avec un lâcher en 1980 (fig. 1). Les observations effectuées au cours des années suivantes ont démontré que le parasite était bien établi dans les deux endroits en 1981 et, que, dès 1984, il s'était

$\overline{0031-9511 / 91} \$ 1.00+.10$ dispersé tout le long de la Vallée du SaintLaurent jusqu'à Montmagny, sise à près de $100 \mathrm{~km}$ à l'est du lieu d'introduction de SaintAugustin-de-Desmaures (Letendre et al. 1986). Afin de connaître l'aire de distribution réelle du D.dryas au Québec, une étude plus complète fut entreprise en 1986.

Au printemps et à l'été de 1986, des échantillons furent prélevés dans des luzernières des 12 régions agricoles du Québec. Les échantillons furent cueillis durant les deux premières semaines de juin et durant les deux semaines de la mi-août, de façon à coïncider avec les périodes d'envol des adultes du $D$. dryas. Les insectes furent récoltés à l'aide d'un filet fauchoir standard de $38 \mathrm{~cm}$ de diamètre, à raison de 100 coups par champ. Selon les régions, de deux à neuf endroits furent échantillonnés, soit un total de 47 endroits dans les 12 régions. Toutes les récoltes furent examinées en laboratoire et classées selon la présence ou l'absence du $D$. dryas. 
Le parasite fut retrouvé dans chacune des 12 régions et à 46 des 47 endroits échantillonnés (fig. 1). Le nombre de captures varie de 1 à 63 individus par 100 coups de filet.

La capture du parasite à Carleton, dans la péninsule de Gaspé, suggère qu'il s'est propagé au rythme d'environ $70 \mathrm{~km}$ par an. Ceciest en accord avec Harcourt et al. (1986) qui ont déterminé que le parasite augmentait son aire de colonisation dans le sud ontarien au taux de $60 \mathrm{~km}$ par an.

Certaines captures sont difficilement explicables par la seule dissémination naturelle du parasite à partir des lieux d'introduction de Saint-Hyacinthe et de Saint-Augustin-deDesmaures. Ainsi, compte tenu du fait que les vents dominants sont d'ouest en est sur la majorité du territoire québecois, la présence du $D$. dryas à l'ouest de Montréal peut être attribuée à une dissémination provenant des lieux d'introduction situés dans la Vallée de l'Outaouais en Ontario où il fut propagé par D.G. Harcourt et J.C. Guppy. D'autre part, la découverte du parasite à Notre-Dame-du-Nord et à Ville-Marie, région 9, résulte vraisemblablement d'un autre lâcher réalisé en 1985, à New Liskeard, dans l'est de l'Ontario.

L'évolution des populations du D.dryas et des dommages causés par l'agromyze dans la parcelle d'introduction de Saint-Augustinde-Desmaures sont présentés au tableau 1. L'évaluation des populations du parasite fut réalisée à l'aide d'un échantillonnage de 100 coups de filet deux fois par semaine durant les périodes d'envol des adultes, tandis que le pourcentage de folioles minées fut obtenu par l'examen de 100 feuilles sur un échantillon constitué de 25 tiges prélevées au hazard deux fois par semaine.

Suite à la capture initiale du parasite en 1981, les populations de ce dernier ont graduellement augmenté durant les deux années suivantes. Toutefois, en 1986 , les populations du $D$. dryas chutaient à des niveaux très bas suite à une baisse importante des populations de l'agromyze. L'effet du parasite s'est fait sentir dès 1984 alors que le nombre de folioles minées par l'agromyze a été réduit presque à néant, ce qui confirme les données de Harcourt et al. (1986) qui démontrent qu'une fois établi, le $D$. dryas prend environ trois ans pour réduire les populations de l'agromyze à des niveaux sous-économiques.

Selon Guppy et al. (1988), le succès du parasite à diminuer les populations d'agromyze de façon si importante et rapide est attribuable à un synchronisme étroit entre le parasite et son hôte. Cette synchronisation est maintenue

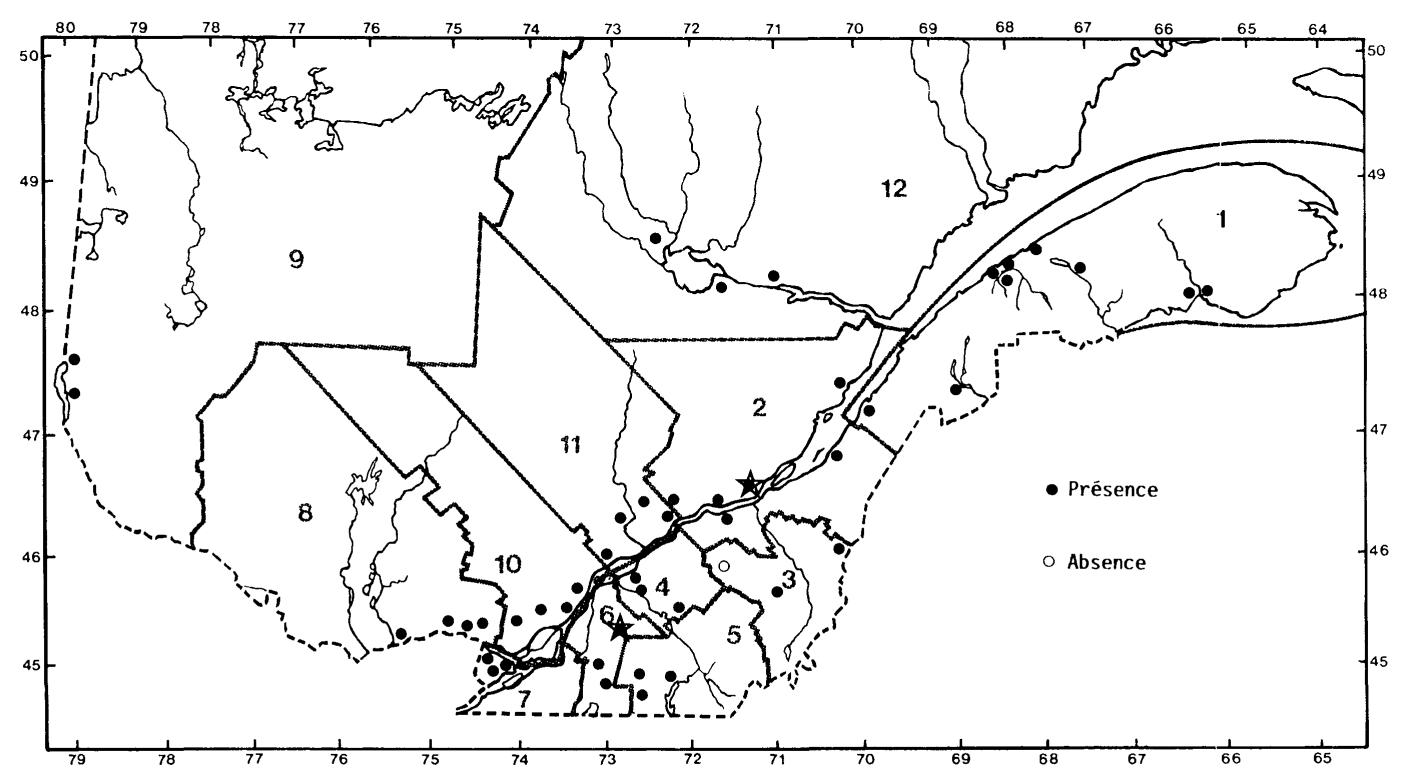

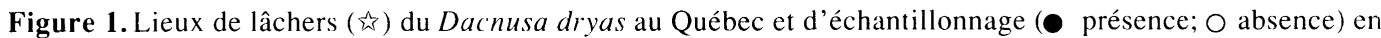
1986. Les régions agricoles sont identifiées par les chiffres 1 à 12 . 
Tableau 1. Populations du Dacnusa dryas et pourcentage de folioles minées par l'Agromyza frontella au lieu d'introduction de Saint-Augustin-de-Desmaures de 1981 à 1986

\begin{tabular}{cccc}
\hline Année & $\begin{array}{c}\text { Regain } \\
\text { de la luzerne }\end{array}$ & $\begin{array}{c}\text { Nombre maximal de } \\
\text { D.dryas par 100 } \\
\text { coups de filet }^{\S}\end{array}$ & $\begin{array}{c}\text { Pourcentage } \\
\text { de folioles minées } \\
\text { sur la luzerne }^{\dagger}\end{array}$ \\
\hline 1981 & 1 & Introduction & 50 \\
1982 & 1 & 1 & 70 \\
1983 & 2 & 1 & 40 \\
1984 & 1 & 1 & 65 \\
& 2 & 62 & 65 \\
1986 & 1 & 96 & 65 \\
& 2 & 88 & 2,5 \\
& 1 & 20 & Trace \\
& 2 & 1 & Trace \\
\hline
\end{tabular}

$\S$ Basé sur des relevés effectués deux fois par semaine durant les périodes d'envol des adultes du $D$. dryas.

$\doteqdot$ Sur 100 feuilles trifoliolées prélevées deux fois par semaine.

d'une année à l'autre grâce au fait que la période d'envol du $D$. dryas coïncide avec la période d'éclosion maximale des oeufs de la première génération d'agromyze.

La réduction des populations d'agromyze à des niveaux très bas soulève des questions quant à la persistance du $D$. dryas en absence d'hôtes secondaires. Toutefois, des observations récentes en Ontario(Meloche et Guppy 1990) démontrent l'existence d'un mécanisme permettant aux populations du parasite de maintenir une masse critique même sous des populations faibles d'agromyze.

Les auteurs remercient Richard Dostie et John Walker pour leur participation lors de la cueillette et de l'examen des échantillons.
Guppy, J.C., F. Meloche et D.G. Harcourt. 1988. Seasonal development, behavior, and host synchrony of Dacnusa dryas (Nixon) (Hymenoptera: Braconidae) parasitizing the alfalfa blotch leafminer, Agromyza frontella (Rondani) (Diptera: Agromyzidae). Can. Entomol. 120: 145-152.

Harcourt, D.G., J.C. Guppy et C.R. Ellis. 1986. Establishment and spread of Dacnusa dryas (Hymenoptera: Braconidae), an exotic parasite of the alfalfa blotch leafminer in Ontario. Proc. Entomol. Soc. Ont. 117: 29-33.

Letendre, M., R. Dostie, D.G. Harcourt, J.C. Guppy et M. O'c. Guibord. 1986. The distribution and impact of Dacnusa dryas (Hymenoptera: Braconidae), an introduced parasite of the alfalfa blotch leafminer, in Quebec. Proc. $23^{\text {rd }}$ N.E. regional alfalfa, corn and small grains Insect Conf. Virginia State Univ., Blacksburg, pp. 31-37.

Meloche, F. et J.C. Guppy. 1990. Encapsulation of eggs and larvae of Dacnusa dryas (Hymenoptera: Braconidae) and its importance in host-parasite coexistence. Environ. Entomol. 19:423-427. 